\title{
Controllability of Nonlinear Systems
}

\author{
JERZY KLAMKA \\ Institute of Automation, \\ Technical University, \\ street Akademicka 16, 44-100 Gliwice, \\ POLAND \\ jklamka@ia.polsl.gliwice.pl
}

Abstract: In the paper, infinite-dimensional, continuous-time control systems described by nonlinear abstract differential equations are considered. Using methods of functional analysis sufficient conditions for constrained exact local controllability are formulated and proved. It is generally assumed that the values of controls are in a convex and closed cone with vertex at zero. Illustrative examples are also given. Moreover, some remarks and comments on controllability problems for nonlinear dynamical systems are presented.

Key-Words: Controllability. Nonlinear systems. Continuous-time systems. Infinite-dimensional systems.

\section{Introduction*}

Controllability is one of the fundamental concept in modern mathematical control theory [5]. Roughly speaking, controllability generally means, that it is possible to steer dynamical system from an arbitrary initial state to an arbitrary final state using the set of admissible controls. In the literature there are many different definitions of controllability which depend on the type of dynamical system [2], [4], [5], [6], [8], [9]. For infinite dimensional dynamical systems it is necessary to distinguish between the notions of approximate and exact controllability [2], [4], [5], [6], [8]. It follows directly from the fact, that in infinitedimensional spaces there exist linear subspaces which are not closed. On the other hand, for nonlinear dynamical systems there exist two fundamental concepts of controllability; namely local controllability and global controllability [1], [4], [7], [10]. Therefore, for nonlinear abstract dynamical systems defined in infinite-dimensional spaces the following four main kinds of controllability are considered: local approximate controllability, global approximate controllability, local exact controllability, and global exact controllability. Controllability problems for finitedimensional nonlinear dynamical systems have been considered in many publications; see [5] for an extensive review of the literature. However, there exist only a few papers on controllability problems for infinite-dimensional nonlinear systems. The present paper is devoted to a study of the local exact controllability with constrained controls for nonlinear

\footnotetext{
* This work was supported by Komitet Badan Naukowych under grant 8T11A 01819
}

stationary infinite-dimensional dynamical systems defined in Banach spaces. For such dynamical systems direct verification of the local exact controllability is rather difficult task. Therefore, using methods of nonlinear functional analysis [1], [2], [3], [6] it is shown that local exact controllability of nonlinear dynamical system can be checked by global exact controllability criteria for appropriate defined associated simplified linear infinite-dimensional dynamical. General results are then applied for verification of local controllability for two finite-dimensional nonlinear dynamical systems. The results presented in the paper extend to a more general class of nonlinear abstract dynamical systems previous controllability theorems given in publications [1], [3].

\section{System Description}

Let us consider the nonlinear infinite-dimensional control system described by the abstract ordinary differential equation

$x^{\prime}(t)=f(x(t), u(t)) \quad$ for $t \in[0, T]$

with zero initial condition $\mathrm{x}(0)=0$, where $\mathrm{f}: \mathrm{X} \times \mathrm{U} \rightarrow$ $X$ is a nonlinear mapping such that $f(0,0)=0$; here $X$ and $U$ are real Banach spaces. It is assumed that the mapping $\mathrm{f}$ is continuously Frechet differentiable with respect to both arguments.

Let $\mathrm{U}_{0} \subset \mathrm{U}$ be a closed convex cone in the space $U$ with vertex at zero and nonempty interior. The set of admissible controls for the system (1) is given by $\mathrm{U}_{\mathrm{ad}}=\mathrm{L}_{\infty}\left([0, \mathrm{~T}], \mathrm{U}_{0}\right)$. For the dynamical system (1) it is 
possible to define many different concepts of controllability [5]. In the sequel we shall focus our attention on so-called constrained exact controllability in a given time interval $[0, T]$. In order to do that, first of all let us introduce the notion of the attainable set at time $\mathrm{T}>0$ from zero initial state $\mathrm{x}(0)=0$, denoted by $\mathrm{K}_{\mathrm{T}}\left(\mathrm{U}_{0}\right)$ and defined as

$\mathrm{K}_{\mathrm{T}}\left(\mathrm{U}_{0}\right)=\left\{\mathrm{x} \in \mathrm{X}: \mathrm{x}=\mathrm{x}(\mathrm{T}, 0, \mathrm{u}), \mathrm{u}(\mathrm{t}) \in \mathrm{U}_{0}\right.$ fora.e. $\left.\mathrm{t} \in[0, \mathrm{~T}]\right\}$

where $x(t, 0, u),(t>0)$ is the unique solution of the equation (2) with zero initial condition and control $u \in$ $\mathrm{U}_{\mathrm{ad}}$. Under the assumptions stated on the nonlinear mapping $f$, such a solution always exists [2]. Using the concept of the attainable set, we may introduce the fundamental definitions of constrained controllability for dynamical system (1) [6].

Definition 2.1 [6] The dynamical system (1) is said to be $\mathrm{U}_{0}$-exactly locally controllable in $[0, \mathrm{~T}]$ if the attainable set $\mathrm{K}_{\mathrm{T}}\left(\mathrm{U}_{0}\right)$ contains a neighbourhood of the origin in the state space $\mathrm{X}$.

For finite-dimensional state space, when $\mathrm{X}=$ $\mathrm{R}^{\mathrm{n}}$ we may omit the word "exact" in the Definition 2.1, since in this case exact controllability is equivalent to approximate controllability [2], [5], [8].

Definition 2.2 [6] System (1) is said to be $\mathrm{U}_{0}$-exactly globally controllable in $[0, T]$ if $\mathrm{K}_{\mathrm{T}}\left(\mathrm{U}_{0}\right)=\mathrm{X}$.

Similarly, as in the previous case, for finitedimensional state space $X=R^{n}$, we may omit the word "exact" in Definition 2.2.

It is convenient to study constrained controllability problems for the nonlinear dynamical system (1) with the aid of its associated linear approximation of the following form

$z^{\prime}(t)=F z(t)+G v(t) \quad$ for $t \in[0, T]$

with zero initial condition $x(0)=0$, where $F: X \rightarrow X$ and $\mathrm{G}: \mathrm{U} \rightarrow \mathrm{X}$ are linear bounded operators given by $\mathrm{F}=\mathrm{D}_{1} \mathrm{f}(\mathrm{x}(\mathrm{t}, 0, \mathrm{u}), \mathrm{u}(\mathrm{t})) \quad \mathrm{G}=\mathrm{D}_{2} \mathrm{f}(\mathrm{x}(\mathrm{t}, 0, \mathrm{u}), \mathrm{u}(\mathrm{t}))$

Here $D_{1} f$ and $D_{2} f$ are the partial Frechet derivatives of the mapping $\mathrm{f}$ with respect to the first and second arguments of function $\mathrm{f}$ respectively.

In what follows we shall concentrate on constrained controllability problems for a special case of nonlinear system (1), namely we shall consider the following infinite-dimensional system [1], [3], [5].
$x^{\prime}(t)=A(x(t)) x(t)+B(x(t)) u(t) \quad$ for $t \in[0, T]$

with zero initial condition $x(0)=0$, where $A$ : $X \rightarrow X$ and $B: U \rightarrow X$ are nonlinear Frechet differentiable operators.

It should be mentioned, that the nonlinear dynamical system (4) satisfies all the assumptions posed on general nonlinear dynamical system (1). Therefore, under the assumptions on nonlinear function $\mathrm{f}$ stated above, the linear approximation of the dynamical system (4) has the following form

$z^{\prime}(t)=A(0) z(t)+B(0) v(t) \quad$ for $t \in[0, T]$

In the next sections using the methods of nonlinear functional analysis we shall formulate and prove sufficient conditions of constrained controllability both for infinite and finite dimensional dynamical systems of the form (4).

\section{Constrained Controllability}

In order to compare constrained controllability results for nonlinear and associated linear system, we need the following general theorem, which is proved in [6].

Theorem 3.1 [6] Suppose that

(i) $\mathrm{f}(0,0)=0$,

(ii) $\mathrm{U}_{0} \subset \mathrm{U}$ is a closed convex cone with vertex at zero,

(iii) The linear dynamical system (3) is $\mathrm{U}_{0}$-exactly globally controllable in $[0, T]$.

Then the nonlinear dynamical system (1) is $\mathrm{U}_{0}$-exactly locally controllable in $[0, \mathrm{~T}]$.

Roughly speaking Theorem 3.1 asserts, that under suitable assumptions posed on nonlinear system (1), if its linear approximation (3) is $\mathrm{U}_{0}$-(exactly) globally controllable, then (1) is locally $\mathrm{U}_{0}$-(exactly) controllable.

In practical applications of Theorem 2.1, the most difficult problem is to verify the assumption (iii) about constrained global controllability of the linear approximation (3). In order to avoid this disadvantage, we may use the following corollary [6].

Corollary 3.1 [6] Suppose that assumptions (i) and (ii) of Theorem 2.1 are satisfied, and that $\mathrm{U}_{0}$ has nonempty interior in the space $\mathrm{U}$. Then the nonlinear dynamical system (1) is $\mathrm{U}_{0}$-exactly locally controllable in $[0, \mathrm{~T}]$ if the associated linear approximation (3) is U-exactly globally controllable in $[0, T]$ (i.e. exactly-globally controllable without any constraints) and

$\operatorname{ker}\left(\mathrm{sI}-\mathrm{F}^{*}\right) \cap\left(\mathrm{GU}_{0}\right)^{0}=\{0\} \quad$ for every $\mathrm{s} \in \mathrm{R}$ 
where $\mathrm{F}^{*}$ denotes the adjoint linear operator, $\mathrm{GU}_{0}$ is the image of the cone $U_{0}$ under the linear operator $G$, and $\left(\mathrm{GU}_{0}\right)^{0}$ is the polar cone for the cone $\mathrm{GU}_{0}$.

Now, using general results stated in Theorem 3.1 and Corollary 3.1, we shall present sufficient conditions for $\mathrm{U}_{0}$-exact local controllability in $[0, \mathrm{~T}]$ for special case of nonlinear dynamical system (4).

Theorem 3.2. Let $\mathrm{U}_{0}$ be a closed convex cone with vertex at zero. Then nonlinear system (4) is $\mathrm{U}_{0}$-exactly locally controllable in $[0, \mathrm{~T}]$, if linear approximation (5) is $\mathrm{U}_{0}$-exactly globally controllable in $[0, \mathrm{~T}]$.

Proof. First of all let us observe, that for the nonlinear dynamical system (4) function $\mathrm{f}(\mathrm{x}, \mathrm{u})=\mathrm{A}(\mathrm{x}) \mathrm{x}+\mathrm{B}(\mathrm{x}) \mathrm{u}$. Therefore, $f(0,0)=0$ and the assumption (i) if Theorem 3.1 is satisfied. The assumptions (ii) and (iii) of Theorem 3.1 is the same as in our theorem. Hence all the assumptions of Theorem 3.1 are satisfied and Theorem 3.2 follows.

Corollary 3.2. Suppose the assumptions of Theorem 3.2 are satisfied, and that the cone $\mathrm{U}_{0}$ has nonempty interior in the space $U$. Then the nonlinear dynamical system (4) is $\mathrm{U}_{0}$-exactly locally controllable in $[0, \mathrm{~T}]$ if the associated linear approximation (2.5) is U-exactly controllable in $[0, \mathrm{~T}]$ (i.e. without any constraints) and $\operatorname{ker}\left(\mathrm{sI}-\mathrm{A}(0)^{*}\right) \cap\left(\mathrm{B}(0) \mathrm{U}_{0}\right)^{0}=\{0\}$ for every $\mathrm{s} \in \mathrm{R}$

Proof. Let us observe that all the assumptions of Corollary 3.1 are satisfied. Moreover, taking into account equation (5) and equality (6) we obtain immediately relation (7). Hence our corollary follows.

It should be mentioned, that in infinitedimensional cases, conditions for exact global controllability of linear dynamical systems which are needed in the above theorems and corollaries are known to be quite a strong requirement [4],[5],[9],[10]. However, for finite-dimensional case these conditions are not so restrictive [5].

Now, using the general results we shall present certain sufficient conditions for $\mathrm{U}_{0}$-local controllability of nonlinear system (4) in finite dimensional case.

Theorem 3.3. Let $X=R^{n}, U=R^{m}$ and $U_{0}$ be a closed convex cone with vertex at zero and nonempty interior in the space $\mathrm{R}^{\mathrm{m}}$. Then the nonlinear dynamical system (4) is $\mathrm{U}_{0^{-}}$locally controllable in $[0, T]$ if

(i) $\operatorname{rank}\left[\mathrm{B}(0)|\mathrm{A}(0) \mathrm{B}(0)| \mathrm{A}(0)^{2} \mathrm{~B}(0)|\ldots| \mathrm{A}(0)^{\mathrm{n}-1} \mathrm{~B}(0)\right]=\mathrm{n}$ (ii) there is no real eigenvector $\mathrm{W} \in \mathrm{R}^{\mathrm{n}}$ for the matrix $\mathrm{A}(0)^{\mathrm{T}}$ satisfying for every $\mathrm{u} \in \mathrm{U}_{0}$ the following inequality $\mathrm{w}^{\mathrm{T}} \mathrm{B}(0) \mathrm{u} \leq 0$.

Proof. Condition (i) means, that linear dynamical system (5) is globally controllable (without any constraints) in any time interval $[0, \mathrm{~T}][5]$. Condition (ii) in finite-dimensional case is equivalent to the relation (7) [9]. Hence, by Corollary 3.3 our theorem follows.

For dynamical systems with scalar control (i.e. $\mathrm{m}=1$ and $\left.\mathrm{B}(\mathrm{x})=\mathrm{b}(\mathrm{x}) \in \mathrm{R}^{\mathrm{n}}\right)$ from Theorem 3.3 we directly obtain the following corollary.

Corollary 3.3. Let $X=R^{n}, U=R$ and $U_{0}=\{u \in R: u \geq 0\}$. Then the nonlinear dynamical system (5) is $\mathrm{U}_{0}$-locally controllable in $[0, \mathrm{~T}]$ if

(i) $\operatorname{det}\left[\mathrm{b}(0)|\mathrm{A}(0) \mathrm{b}(0)| \mathrm{A}(0)^{2} \mathrm{~b}(0)|\ldots| \mathrm{A}(0)^{\mathrm{n}-1} \mathrm{~b}(0)\right] \neq 0$

(ii) matrix $\mathrm{A}(0)$ has only complex eigenvalues.

Proof. Let us observe, that the positive cone $\mathrm{U}_{0}$ has nonempty interior in the space $\mathrm{R}$. Moreover, when $\mathrm{m}=1$ and $\mathrm{B}(\mathrm{x})=\mathrm{b}(\mathrm{x}) \in \mathrm{R}^{\mathrm{n}}$, conditions (i) in Theorem 3.3 and Corollary 3.4 are of course equivalent. Finally, since w and $-\mathrm{w}$ are both eigenvectors of the matrix $\mathrm{A}(0)^{\mathrm{T}}$ and $\mathrm{u}$ $\geq 0$, then one of the inequalities $\mathrm{w}^{\mathrm{T}} \mathrm{b}(0) \mathrm{u} \leq 0$ or $\mathrm{w}^{\mathrm{T}} \mathrm{b}(0) \mathrm{u} \leq 0$ is false. Therefore, matrix $\mathrm{A}(0)^{\mathrm{T}}$ should have only complex eigenvalues. Hence our corollary follows.

\section{Examples}

In this section we shall present two simple numerical finite-dimensional examples, which illustrate theoretical considerations given in the previous section.

Example 1. Let us consider the following nonlinear finite-dimensional stationary dynamical system with nonnegative controls.

$$
\begin{aligned}
\mathrm{x}_{1}^{\prime}(\mathrm{t})= & 2 \mathrm{x}_{1}(\mathrm{t}) \cos \mathrm{x}_{2}(\mathrm{t})+\mathrm{x}_{2}(\mathrm{t}) \cos \mathrm{x}_{1}(\mathrm{t})+\mathrm{u}_{1}(\mathrm{t}) \operatorname{expx}_{2}(\mathrm{t})- \\
& -\mathrm{u}_{2}(\mathrm{t}) \operatorname{expx}_{1}(\mathrm{t}) \\
\mathrm{x}_{2}^{\prime}(\mathrm{t})= & \mathrm{x}_{1}(\mathrm{t}) \operatorname{expx}_{1}(\mathrm{t})+2 \mathrm{x}_{2}(\mathrm{t}) \operatorname{expx}_{2}(\mathrm{t})+2 \mathrm{u}_{1}(\mathrm{t}) \cos \mathrm{x}_{2}(\mathrm{t}) \\
& -3 \mathrm{u}_{2}(\mathrm{t}) \cos \mathrm{x}_{1}(\mathrm{t})
\end{aligned}
$$

In this case $\mathrm{X}=\mathrm{R}^{2}, \mathrm{U}=\mathrm{R}^{2}$, and the nonnegative cone $\mathrm{U}_{0}=\left\{\mathrm{u}=\left[\mathrm{u}_{1}, \mathrm{u}_{2}\right] \in \mathrm{R}^{2}: \mathrm{u}_{1} \geq 0, \mathrm{u}_{2} \geq 0\right\}$. Moreover, the matrices $\mathrm{A}(\mathrm{x})$ and $\mathrm{B}(\mathrm{x})$ have the following form

$$
A(x)=\left|\begin{array}{cc}
2 \cos x_{2} & \cos x_{1} \\
\exp x_{1} & 2 \exp x_{2}
\end{array}\right| \quad B(x)=\left|\begin{array}{cc}
\exp x_{2} & -\exp x_{1} \\
2 \cos x_{2} & -3 \cos x_{1}
\end{array}\right|
$$

Hence,

$$
A(0)=\left|\begin{array}{ll}
2 & 1 \\
1 & 2
\end{array}\right| \quad \text { and } \quad B(0)=\left|\begin{array}{ll}
1 & -1 \\
2 & -3
\end{array}\right|
$$


Since the matrix $\mathrm{B}(0)$ is nonsingular, condition (i) in Theorem 3.3 is automatically satisfied.

Now, we shall verify condition (ii) of the Theorem 3.3. First of all let us observe, that matrix $A(0)$ has two real eigenvalues, namely; $s_{1}=1$ and $s_{2}=3$. The corresponding eigenvectors are; $\mathrm{w}_{1}=\mathrm{k}_{1}[1,-1]^{\mathrm{T}}$ and $\mathrm{w}_{2}$ $=\mathrm{k}_{2}[1,1]^{\mathrm{T}}$, where $\mathrm{k}_{1} \in \mathrm{R}$ and $\mathrm{k}_{2} \in \mathrm{R}$ are given constants. Therefore, we have

$\mathrm{k}_{1} \mathrm{w}_{1} \mathrm{~B}(0) \mathrm{u}=\mathrm{k}_{1}\left(-\mathrm{u}_{1}+2 \mathrm{u}_{2}\right) \quad \mathrm{k}_{2} \mathrm{w}_{2} \mathrm{~B}(0) \mathrm{u}=\mathrm{k}_{2}\left(3 \mathrm{u}_{1}-4 \mathrm{u}_{2}\right)$ and assumption (ii) in Theorem 3.3 is satisfied (for a given $k_{1}$ and $k_{2}$, the right hand sides of two above equalities may change the sign). Hence, the nonlinear dynamical system (8) is $\mathrm{U}_{0}$-locally controllable in any time interval $[0, \mathrm{~T}]$.

Example 2. Let us consider nonlinear finitedimensional stationary dynamical system with the same matrix $\mathrm{A}(\mathrm{x})$ as in Example 1, but with the matrix $\mathrm{B}(\mathrm{x})$ given by the following equality

$B(x)=\left|\begin{array}{cc}\exp x_{2} & -\exp x_{1} \\ 2 \cos x_{2} & 3 \cos x_{1}\end{array}\right|$

In this case we have $\quad B(0)=\left|\begin{array}{cc}1 & -1 \\ 2 & 3\end{array}\right|$

$\mathrm{k}_{1} \mathrm{w}_{1} \mathrm{~B}(0) \mathrm{u}=\mathrm{k}_{1}\left(-\mathrm{u}_{1}-2 \mathrm{u}_{2}\right), \quad \mathrm{k}_{2} \mathrm{w}_{2} \mathrm{~B}(0) \mathrm{u}=\mathrm{k}_{2}\left(3 \mathrm{u}_{1}+2 \mathrm{u}_{2}\right)$

Hence, both two above relations do not satisfy the condition (ii) in Theorem 3.3 (for a given $\mathrm{k}_{1}$ and $\mathrm{k}_{2}$, the right hand sides of the above equalities cannot change the sign). Therefore, in this case the nonlinear systems is not $\mathrm{U}_{0}$-locally controllable in any time interval $[0, \mathrm{~T}]$.

\section{Conclusion}

In the present paper exact controllability problems for nonlinear stationary dynamical systems have been considered. Using certain general theorem on constrained exact controllability, sufficient conditions for $\mathrm{U}_{0}$-exact controllability in a given time interval have been formulated and proved both for infinitedimensional and finite-dimensional nonlinear control systems. It is generally assumed, that $\mathrm{U}_{0}$ is a closed convex cone with vertex at zero. Moreover, two illustrative finite-dimensional examples have been presented. The obtained theoretical results extend to more general class of dynamical systems previous results on nonlinear controllability known in the literature. Finally, it should be pointed out, that quite similar methods of functional analysis can be applied for investigation of exact controllability problems in nonlinear nonstationary infinite-dimensional and finitedimensional dynamical systems including dynamical systems with delays.

\section{References:}

[1] N. Carmichael, and M.D. Quinn, Fixed-point methods in nonlinear control, IMA Journal of Mathematical Control and Information, Vol.5, No.1, 1988, pp.41-67.

[2] E.Chukwu,and S.Lenhart,Controllability questions for nonlinear systems in abstract spaces, $J$. Optimization Theory and Applications, Vol.68, No.3, 1991, pp.437-462.

[3] V. Dannon, and A. Kartsatos, The controllability of a quasilinear functional differential system, Annales Polonici Mathematici, Vol. XLVII, 1987, pp.371380

[4] H.O. Fattorini, Local controllability of nonlinear wave equation, Mathematical Systems Theory, Vol.9, No.1, 1975, pp.30-45.

[5] J. Klamka, Controllability of Dynamical Systems, Dordrecht: Kluwer Academic Publishers, 1991.

[6] J. Klamka, Constrained controllability of nonlinear systems, Journal of Mathematical Analysis and Applications, Vol.201, No.2, 1996, pp.365-374.

[7] G. Peichl, and W. Schappacher, Constrained controllability in Banach spaces, SIAM Journal Control and Optimization, Vol.24, No.6, 1986, pp.1261-1275.

[8] T. Schanbacher, Aspects of positivity in control theory, SIAM Journal Control and Optimization, Vol.27, No.3, 1989, pp.457-475.

[9] N.K. Son, A unified approach to constrained approximate controllability for the heat equations and the retarded equations, Journal of Mathematical Analysis and Applications, Vol.150, No.1, 1990, pp.1-19.

[10]H.X. Zhou, Controllability properties of linear and semilinear abstract control systems, SIAM Journal Control and Optimization, Vol. 22, No.3, 1984, pp.405-422. 